Research Article

\title{
A New Method for Artificial Core Reconstruction of a Fracture-Control Matrix Unit
}

\author{
Qiang Liu, ${ }^{1}$ Jianjun Liu $\mathbb{D}^{1,2}{ }^{1,2}$ Guihong Pei, ${ }^{3}{\text { Zhengwen } \mathrm{Zhu}^{1}{ }^{1} \text { and Yun Lei }}^{4,5}$ \\ ${ }^{1}$ School of Geoscience and Technology, Southwest Petroleum University, Chengdu 610500, China \\ ${ }^{2}$ State Key Laboratory of Geomechanics and Geotechnical Engineering, Institute of Rock and Soil Mechanics, \\ Chinese Academy of Sciences, Wuhan 430071, China \\ ${ }^{3}$ School of Architectural Economic and Engineering Management, Hubei Business College, Wuhan 430074, China \\ ${ }^{4}$ Shenyang Research Institute, China Coal Technology \& Engineering Group Corp., Fushun 113122, China \\ ${ }^{5}$ State Key Laboratory of Coal Mine Safety Technology, Fushun 113122, China
}

Correspondence should be addressed to Jianjun Liu; jjliu@whrsm.ac.cn

Received 24 August 2019; Accepted 25 April 2020; Published 25 May 2020

Academic Editor: Timo Saksala

Copyright (c) 2020 Qiang Liu et al. This is an open access article distributed under the Creative Commons Attribution License, which permits unrestricted use, distribution, and reproduction in any medium, provided the original work is properly cited.

The fracture-control matrix unit (F-CMU) is a special body present in low-permeability fractured reservoirs that can be distinguished by a fracture system and a matrix system. The imbibition phenomenon of the F-CMU provides the possibility for secondary development of low-permeability fractured reservoirs because of the driving force including capillary force and gravity. However, the F-CMU is difficult to obtain during the field core drilling, which has limited the development for laboratory dynamic imbibition tests. Therefore, a new F-CMU reconstruction method is proposed in this study. According to the geometry and parameters, combining laser engraving technology, the fracture system is designed and engraved. Then, the F-CMU is established using a three-dimensional (3D) printed material called polyvinyl alcohol (PVA) as fracture support material which has a faster dissolution rate and causes less damage to the core due to water being the solvent. Finally, the porosity, permeability, and wettability of the matrix system and the $\mathrm{T}_{2}$ spectra from nuclear magnetic resonance (NMR) before and after reconstruction are measured. In addition, numerical simulation calculation of F-CMU permeability is performed. The results show that the characteristic parameters of the matrix system hardly change, indicating low damage to the core. The reconstructed fracture system is found on the $\mathrm{T}_{2}$ spectra, and the fracture permeability is consistent by comparing with the experimental and numerical simulation results. The permeability of the fracture system is about $10^{4}$ orders of magnitude of the matrix system, which is closer to real core and meets the requirements needed for dynamic permeability experiments.

\section{Introduction}

With the increase of world energy demand [1], residual oil recovery in the middle and late stages of a low-permeability fractured reservoir is a key and difficult point in on-site oil exploration and development. A large amount of crude oil remains in the matrix, which is difficult to extract by conventional methods. Different permeability characteristics exist between the fracture and matrix system, which lead to fast flow of the injected water along the fracture with high permeability. Eventually, water breaks through and causes a flooding to the producing well. Therefore, the production of the oil field is restricted and the economic benefit is directly affected. However, the special imbibition phenomenon where the injected water in the fracture will be driven by capillary force and gravity into the matrix to replace the residual oil has allowed the possibility for secondary development in the low-permeability fractured reservoir [2-4]. The core containing both the fracture and matrix systems is defined as the fracture-control matrix unit (F-CMU). The laboratory research of the F-CMU is an important way to determine the imbibition process, the influencing factors, and the final oil-water distribution. However, it is almost impossible to get a F-CMU when drilling core in the field, which limits the development of imbibition laboratory tests. Therefore, the core 
reconstruction of the F-CMU is an urgent problem needing to be solved.

To date, researchers had developed different methods and techniques to reproduce cores using conventional ways. These methods can be roughly divided into the following four categories: (a) the parallel plate method, (b) splitting reconstruction, (c) chemical solvent dissolution method, and (d) the 3D printer method. In 1958, the fracture was made by two parallel boards with four adjusting screws to adjust fracture widths [5]. Later, Mattax and Kyte [6] determined that the open faces of two cores were separated by a small metal spacer, so the space between the cores represented a fracture. In 1977, artificially fractured cores were prepared by cutting cylindrical cores along the long axis, and the two halves were held together and the experiments were conducted using a horizontal fracture [7]. After that, using a model similar to Graham and Richardson [5], Bourbiaux and Kalaydjian [8] and Schechter et al. [9] studied the factor effects of imbibition. The above methods have been widely applied to core fracture reconstruction [10-16]. However, these traditional methods have some limitations related to the shape of the fracture and the restoration of the authenticity of the underground reservoir. In the case of the parallel plate method, although the aperture of the fracture can be adjusted, the fracture is not present inside the matrix. On the other hand, for splitting reconstruction, the fracture formed was single, and the shape was uncontrollable. In addition, it was difficult to produce the fracture net or the shape needed for the experiment, which greatly limits the usability of the reconstructed core.

In order to overcome these limitations, the chemical solvent dissolution method was developed, which produced synthetic porous sandstones made by sand and epoxy resin [17-19]. After that, Tillotson et al. [20-22] improved the method, and they reproduced the core using high temperatures and a mixture of sand, kaolinite, and sodium silicate instead of resin. In 2017, Santos et al. [23] developed a new methodology for fractured sample construction. In order to create the fracture, layers of the matrix mixture (clean sand and cement) and fracture support materials (Styrofoam) were laid alternately into a mold, and the model was compacted. After that, the sample models were immersed into paint thinner to dissolve the Styrofoam cuts, leaving out squared or penny-shaped voids that represent fractures and cracks. However, these methods also present some disadvantages. First, the matrix material is made of sand, kaolinite, sodium silicate, or clean sand and cement. These materials not only change the physical properties of the matrix surface but also do not match to the composition of the real rock. Second, chemical solvents such as acids can chemically react with substances in the matrix causing severe damage to the core. Nevertheless, with the development of digital cores [1, 24, 25], laser etching and 3D printing technology [26] were commonly used in core reconstruction $[27,28]$. The advantage is that we can reconstruct the fracture network inside the core so that it can more accurately represent the fracture structure of the actual core. Meanwhile, fracture proppant has a faster dissolution rate and is core friendly. The disadvantage is that the matrix material used in laser etching and 3D printing reconstruction must utilize a dedicated printing material, losing the authenticity of the matrix.
In this paper, we propose a new, efficient, feasible, and low-damage construction method that combines laser engraving and $3 \mathrm{D}$ printer technology, where a F-CMU is reproduced. As much as possible, all of the reconstruction process operations are conducted on real cores to ensure that the composition and physical properties of the core matrix do not change. Meanwhile, the geometry, spatial location, and permeability characteristics of the constructed fracture system can be controlled and designed. Moreover, water is used as a solvent for the $3 \mathrm{D}$ printing materials, as it causes almost no damage to the reconstructed core samples. To verify the correctness of this reconstruction method, we measure the porosity, permeability, and wettability of the matrix system and the NMR $\mathrm{T}_{2}$ spectra of the core samples before and after reconstruction. In addition, numerical simulation and laboratory testing of the fracture system permeability are also performed. The proposed reconstruction method provides useful help for laboratory experiments at the core scale in lowpermeability fractured reservoirs.

\section{Materials and Methods}

First, the meaning of F-CMU is explained in Section 2.1. After that, the core samples and material requested are prepared. Finally, the process of reconstruction is described in detail. In addition, we also introduced experimental apparatus and NMR analysis method. Figure 1 shows the sequence of steps adopted for the reconstructed process. The techniques implemented in each of these steps are described in detail in the following section.

2.1. Fracture-Control Matrix Unit. It has become an effective means to exploit low-permeability fractured reservoirs by adopting hydraulic fracturing technology. After fracturing, natural and artificial fractures coexist in the reservoir. There are two important systems in the low-permeability fractured reservoir, namely, the fracture system and the matrix system. The fracture system is characterized by high permeability and a low porosity that can be considered to be zero, whereas the matrix system is characterized by low permeability and high porosity. In the previous literature, there is no clear definition used to describe the fracture and matrix system. This limits the understanding of fractured reservoirs.

Therefore, on the basis of summarizing previous studies, we propose using a F-CMU. It should be used in lowpermeability fractured reservoirs, especially for artificial hydraulic fracturing reservoirs, where a specific unit body contains both the fracture system (natural and artificial fracture) and a matrix system. The main characteristics of this unit are as follows: (a) the permeability of the fracture system is significantly different from the matrix system, (b) the fluid exchanges between the two systems through the seepage, and (c) the range of the seepage area controlled by different fractures is different. The schematic diagram of the F-CMU is shown in Figure 2.

2.2. Experimental Apparatus. A rock cutting equipment (Model TXDQ-1T, ZHONGKE, China) is used to split the core into two equal halves. The machine is controlled by 


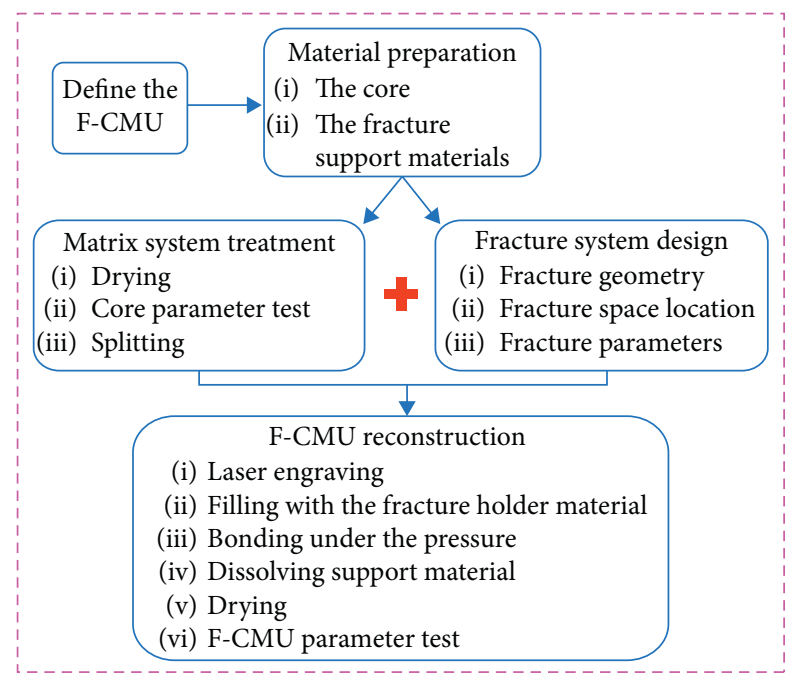

Figure 1: F-CMU core reconstruction workflow.

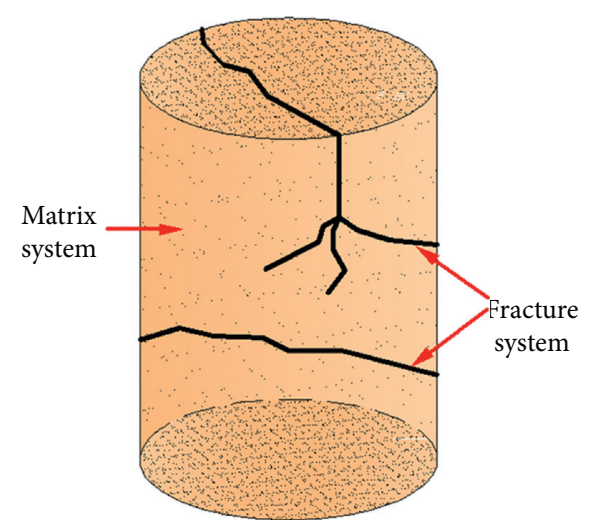

FIgURE 2: Fracture-control matrix unit schematic.

computer program, and it is equipped with high-precision walking system. A laser engraving machine (Model A18, Ruizhi, China) is mainly used to fracture system carving, with necessary instruments included. The engraving process is automatic with an accuracy of $0.001 \mathrm{~mm}$. The core analysis system (Model CMS-300, Temco, America) is used to measure core porosity and permeability before and after reconfiguration. The core nuclear magnetic resonance analysis system (NINMAG, China) is Model Animr-150 with a magnetic field intensity of $0.23 t \pm 0.03$, a maximum echo number of 8000 , a minimum echo interval below 150 $\mu s$, and a minimum digital acquisition interval of $50 \mathrm{~ns}$. The interface parameter integrated measurement system (Model DSA30S, KRUSS, Germany) is used to test the wettability.

2.3. Material Preparation. Two core samples drilled from the Xinzhao district of the Daqing Oil field is adopted, with core depths of $1424.31-1429.50 \mathrm{~m}$ and $1393.50-1402.99 \mathrm{~m}$, and the core samples are numbered 85-4 and 24-2, respectively, as shown in Figure 3. The block is a typical low-permeability fractured reservoir, and the basic parameters of the core samples are tested as shown in Table 1.

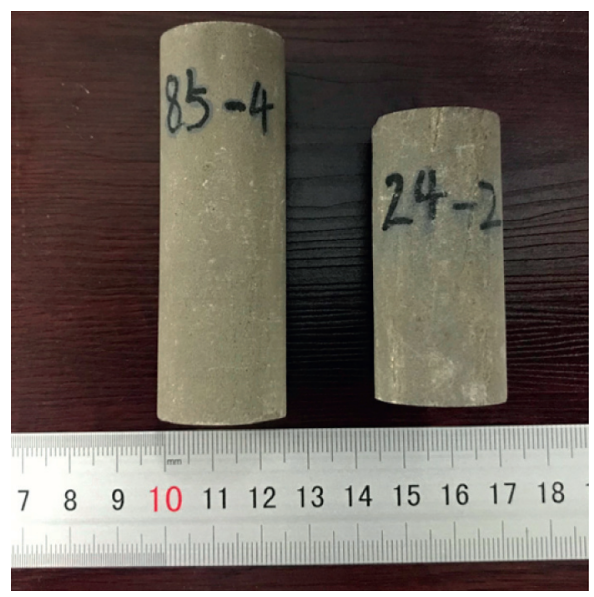

Figure 3: Core samples.

TABLE 1: Basic parameters of the core samples.

\begin{tabular}{lcc}
\hline ID of samples & Diameter $(\mathrm{cm})$ & Length $(\mathrm{cm})$ \\
\hline $85-4$ & 2.52 & 7.53 \\
$24-2$ & 2.50 & 5.59 \\
\hline
\end{tabular}

In this study, PVA is used as a fracture support material during core compression bonding. It has the following advantages: (1) good water solubility, (2) a very fast dissolution rate, and (3) it does not chemically react with the matrix by using water as a solvent. These advantages ensure that the physical properties of the core are not changed.

The dissolution rate of the PVA is tested before the experiment. The PVA is put into a beaker, and water of different temperatures $\left(30^{\circ} \mathrm{C}, 60^{\circ} \mathrm{C}\right.$, and $\left.80^{\circ} \mathrm{C}\right)$ is added, as shown in Figure 4. We observed that the PVA material began to soften, the flocculent peeled off, and it finally becomes very soft and breakable over time. The dissolution proportion curve at different water temperatures is calculated by the mass method, as shown in Figure 5.

From Figure 4, the PVA material first absorbs water and then swells. After a period of time, the surface of the material begins to dissolve, and flocculent obviously peels off. Finally, the material dissolves into flocculent particles. As shown in Figure 5, temperature directly affects the dissolution rate. The dissolution rate is highest at $80^{\circ} \mathrm{C}$, followed by $60^{\circ} \mathrm{C}$, and it is at the lowest at $30^{\circ} \mathrm{C}$. However, the dissolution rate at $60^{\circ} \mathrm{C}$ and $80^{\circ} \mathrm{C}$ is not much different. In addition, it is found that the temperature has a great influence on the initial dissolution rate of the PVA material. Therefore, considering the convenience of the operation of the F-CMU core reconstruction process, we saturate the core with water at $60^{\circ} \mathrm{C}$ for PVA dissolution.

The core samples are washed and dried before reconstitution. Then, the porosity, permeability, and wettability of matrix are measured. After that, core samples are saturated with water, and NMR $T_{2}$ spectra tests are performed.

2.4. F-CMU Core Reconstruction. Before the core reconstruction experiment, the fracture system was designed using laser engraving model software. Since fractal propagation exists widely in hydraulic fracture network, a great 

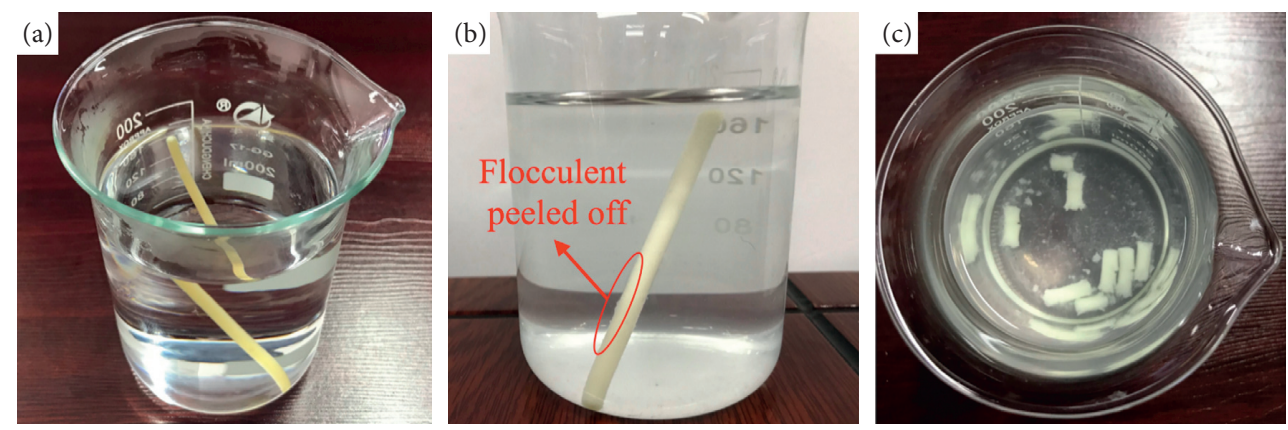

Figure 4: PVA water soluble material dissolution process. (a) Initial state of solution. (b) Flocculent peeled off on the surface. (c) Final state of solution.

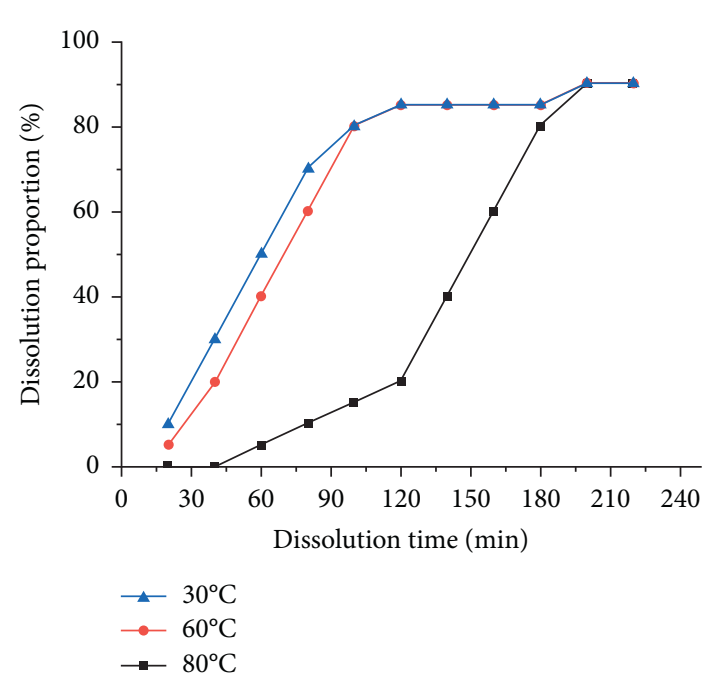

FIgURE 5: Dissolution proportion curve.

number of investigations indicated that the fracture morphology of hydraulic fracturing is similar to the fractal-like tree branching model and bending model [29-34]. Thus, the core reconstruction method proposed in this paper is different from the traditional single straight fracture, and the bifurcation fracture (Y-type) and bending fracture (S-type) are adopted based on the fractal characteristics of the hydraulic fracture. Regarding fracture structure parameters, the bifurcation angle of the Y-type fracture and the tortuosity of the S-type fracture are not fixed parameters. The selected values are to fill the core cross section. In addition, for the width and aperture of the fracture, refer to the field data of Daqing Oil field [35]. The parameters and structure are shown in Table 2 and Figures 6(a)-6(c).

Then, the physical parameters of cores are tested. After that, the core samples (85-4 and 24-2) are split into two equal halves, as shown in Figure 7(a). The PVA material is cut into thin slices for fracture support.

After that, we use a laser engraving machine to engrave the cores, as shown in Figure 7(b). In order to reduce the damage, only one side of the core is engraved. Then, the fracture is filled with the prepared PVA support material to avoid damaging the engraved fracture during compression bonding (Figure $8(\mathrm{a})$ ). Later, the adhesive is smeared along the edge of the core. Finally, the cores are wrapped with a heat shrinkable tube (Figure 8(b)) and placed under conditions of $1 \mathrm{MPa}$ for 72 hours.

In the final step, the bonded cores are saturated with distilled water and then immersed into a constant temperature water bath at $60^{\circ} \mathrm{C}$. The PVA material began to dissolve and left out the PVA fracture support material completely. Finally, the cores are dried to obtain a reconstituted F-CMU. The reconstituted F-CMU and the front and the ends of the F-CMU core are shown in Figures 9(a)-9(c), respectively.

After the reconstruction of the F-CMU, the physical parameters of core samples are tested again, including the matrix porosity, permeability, and wettability, as well as the fracture permeability. Then, the core samples are saturated with water, and the NMR $\mathrm{T}_{2}$ spectra test is also performed.

2.5. NMR Analysis Method. The basic principle of nuclear magnetic resonance analysis technology is to analyze the pore structure by measuring the magnetic resonance signal intensity of the hydrogen nuclei $\left({ }^{1} \mathrm{H}\right)$ in the rock pore fluid and the interaction between the fluid and the solid surface of the rock pore. When the fluid is confined in the pore, the NMR relaxation time is determined by the pore size due to the interaction between the fluid and the pore wall. The larger the pore, the longer the relaxation time [36].

In this paper, the first NMR test is performed when the original cores (85-4 and 24-2) are saturated with water. The relationship curve of relaxation time and the signal intensity (porosity component) calculated by computer is obtained. After the F-CMU is reconstructed, the second test is conducted and the relationship curve is obtained. The results of the two NMR tests are compared.

\section{Results and Discussion}

Matrix porosity, permeability, and surface wettability are important basic parameters for evaluating its imbibition capacity [37]. Therefore, in the process of core reconstruction of the F-CMU, it is extremely important to ensure the consistency of the matrix porosity, permeability, and surface wettability with the original core. In this section, the test data of the two core samples, including the change of matrix properties before and after reconstitution, the permeability of the Y-type and S-type fractures, and the pore 
TABLE 2: Fracture parameters of the core samples.

\begin{tabular}{lccccc}
\hline Fracture shape & ID of samples & Bifurcation angle $\left(^{\circ}\right)$ & Tortuosity (dimensionless) & Width $(\mathrm{mm})$ & Aperture $(\mathrm{mm})$ \\
\hline Y-shape fracture & $85-4$ & 25 & - & 3.0 & 0.5 \\
S-shape fracture & $24-2$ & - & 1.131 & 4.2 & 0.5 \\
\hline
\end{tabular}

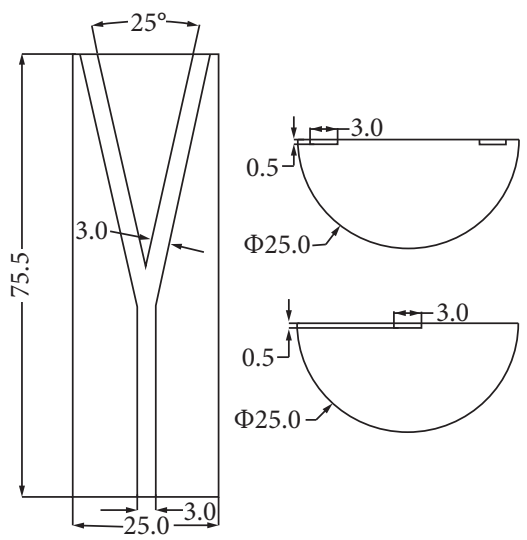

(a)

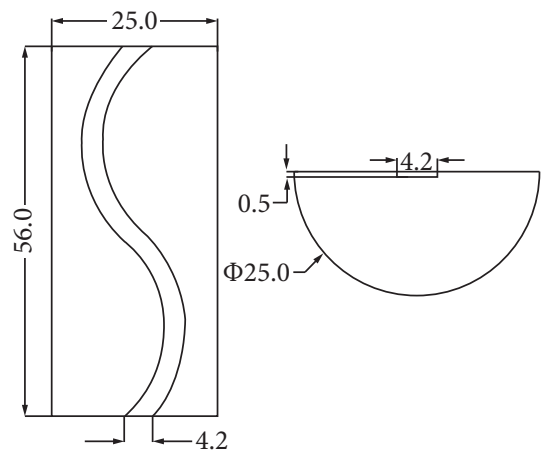

(b)

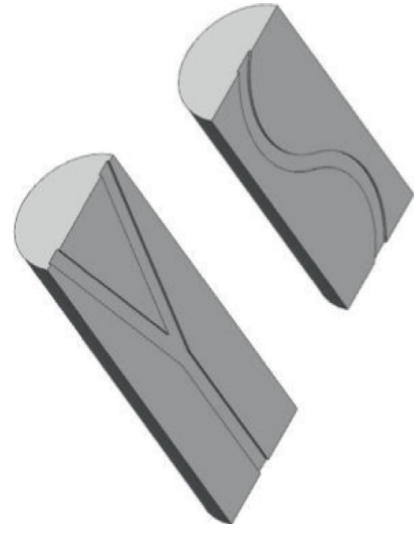

(c)

FIGURE 6: Parameters and 3D structure of the Y-shaped fracture and S-shaped fracture. (a) Y-shaped fracture parameters. (b) S-shaped fracture parameters. (c) 3D structure of two fractures (unit: $\mathrm{mm}$ ).
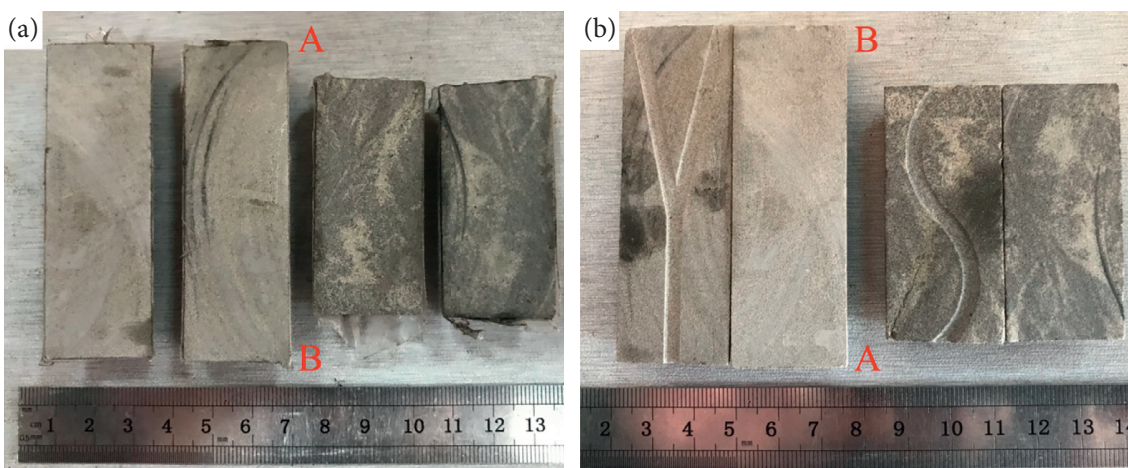

FIGURE 7: F-CMU core laser engraving process. (a) Core samples were split into two equal halves. (b) Y-shaped fracture and S-shaped fracture laser engraving.
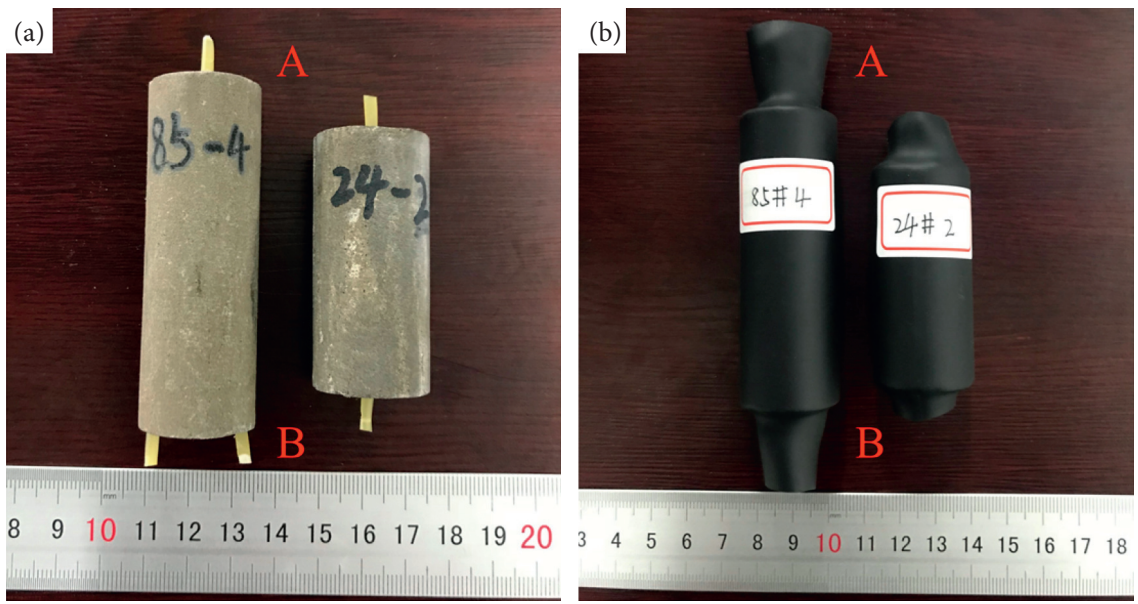

FIGURE 8: F-CMU core bonding process. (a) Filled with the PVA support material. (b) Wrapped with heat shrinkable tube and bonding under the pressure. 

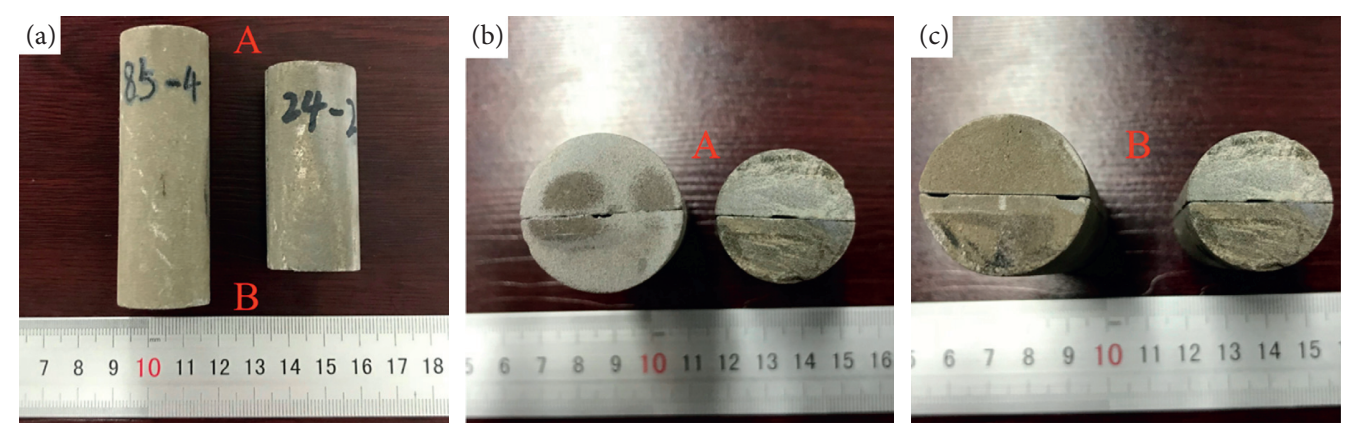

Figure 9: F-CMU reconstituted core. (a) Front of the core. (b) A end face of core. (c) B end face of core.

size and distribution, are compared and analyzed to verify the correctness of the reconstruction method.

3.1. Matrix Porosity and Permeability. The porosity and permeability of the core have great influence on the fluid flow $[38,39]$. When the F-CMU core is reconstructed, the matrix porosity and permeability of the F-CMU cores are measured, and the test results are shown in Table 3. CMS300 computer-controlled core overburden pressure porosity and permeability tester adopt the Darcy-Klinkenberg comprehensive calculation model and an improved Boyle's law to calculate the porosity and permeability of low-permeability core samples. The distribution of gas pressure along the sample length varies with both position and time. In the process of computing, Klinkenberg gas slippage effect has been considered [40, 41].

First, compared with other reconstruction methods, the core structure is maintained as large as possible, and the mineral composition is not altered. The core samples are processed by only splitting, engraving, and bonding. Second, the binder (specially for rock) and the solvent (water) that we use do not chemically react with the matrix. On the one hand, there is no chemical damage to the matrix, which leads to changes in the matrix composition. It also does not cause any change to the pore structure inside the matrix.

As can be seen from Table 3, the matrix porosity of core $85-4$ and core $24-2$ before reconstruction is $18.33 \%$ and $12.97 \%$, respectively. After reconstitution, the porosity is only $17.89 \%$ and $12.01 \%$, respectively. The magnitude of change is $0.024 \%$ and $0.074 \%$, respectively. We know that even if two identical cores have small changes during the test, it is due to temperature differences and operational factors. Moreover, during the compaction process of reconstruction, the pores of the core will become slightly smaller under confining pressure, which will also cause a slight decrease in the porosity after reconstruction.

Regarding permeability, the matrix permeability of core 854 and core $24-2$ is $2.96 \mathrm{mD}$ and $0.85 \mathrm{mD}$ before reconstruction. After reconstitution, they are $2.84 \mathrm{mD}$ and $0.80 \mathrm{mD}$, respectively. The magnitude of change is $0.04 \%$ and $0.05 \%$, respectively. The matrix permeability is mainly affected by matrix pore connectivity. In the process of reconstruction, the porosity of the matrix decreased slightly. At the pore scale, it is indicated that some pores are compressed and closed. Furthermore, the argillaceous sandstone is dissolved, leading to the pores
TABLE 3: Porosity and permeability of the core matrix.

\begin{tabular}{lccc}
\hline Test status & $\begin{array}{c}\text { ID of } \\
\text { samples }\end{array}$ & $\begin{array}{c}\text { Porosity } \\
(\%)\end{array}$ & $\begin{array}{c}\text { Permeability } \\
(\mathrm{mD})\end{array}$ \\
\hline Before & $85-4$ & 18.33 & 2.96 \\
reconstitution & $24-2$ & 12.97 & 0.85 \\
After & $85-4$ & 17.89 & 2.84 \\
reconstitution & $24-2$ & 12.01 & 0.80 \\
\hline
\end{tabular}

becoming blocked by water saturation. This causes a slight decrease in matrix permeability after reconstruction. However, small changes in matrix porosity and permeability are acceptable before and after reconstitution.

3.2. Matrix Surface Wettability. The wettability of the matrix surface is the fundamental condition for imbibition for the F-CMU. Wettability is characterized by the contact angle. When $\theta>90^{\circ}$, the core is oil-wetted, and when $\theta<90^{\circ}$, the core is water-wetted [42]. The wettability is directly affected by the mineral composition of the core matrix. In this study, the surface structure and mineral composition of the core are not destroyed.

The measured values of the contact angle of the cores before and after reconstitution are shown in Figure 10.

Figure 10 shows that the contact angles of core 85-4 and 24-2 are $14.87^{\circ}$ and $21.09^{\circ}$ before the reconstruction, respectively, and after reconstruction, they are $14.90^{\circ}$ and $21.10^{\circ}$, respectively. The surface wettability hardly changed, as the surface of the core matrix is not destroyed during the reconstruction process.

3.3. NMR $T_{2}$ Spectra. The NMR $T_{2}$ spectra test is performed before and after reconstruction, and the results are shown in Figure 11.

As shown in Figures 11(a) and 11(b), the $T_{2}$ spectra of the two cores have the same trend and have multiple peaks before the reconstruction, indicating that the pore distribution of cores $85-4$ and 24-2 is not uniform. Peaks No. 1 , No. 2, and No. 3 from core 85-4 appear near $100 \mu \mathrm{s}$, $1000-10000 \mu \mathrm{s}$, and $10000-100000 \mu \mathrm{s}$, respectively, indicating that there are nanoscale pores, micropores, and mesopores inside it. In addition, peak No. $1<<$ No. $2<$ No. 3 , indicating that the micropores and mesopores in core 854 are developed and that there are a small number of nanoscale pores. Peaks No. 1, No. 2, and No. 3 of core $24-2$ appear near $100 \mu \mathrm{s}, \quad 1000-10000 \mu \mathrm{s}$, and 


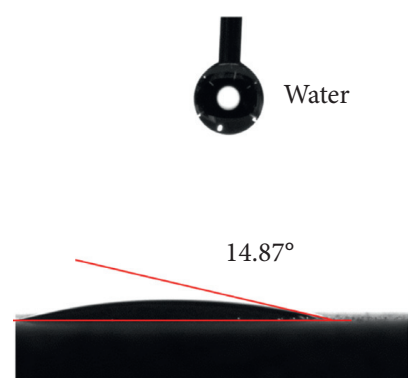

(a)

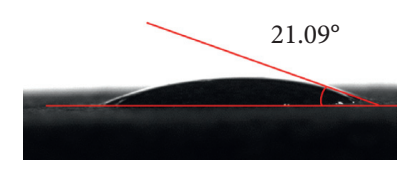

(b)
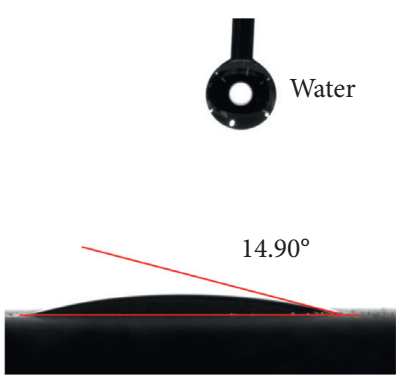

(c)

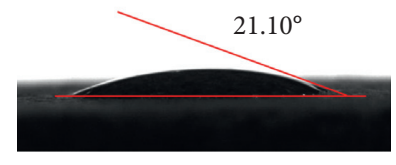

(d)

Figure 10: The contact angles of the core samples. (a, b) Contact angles of core 85-4 and core 24-2 before reconstruction. (c, d) Contact angles of core $85-4$ and core $24-2$ after reconstruction.

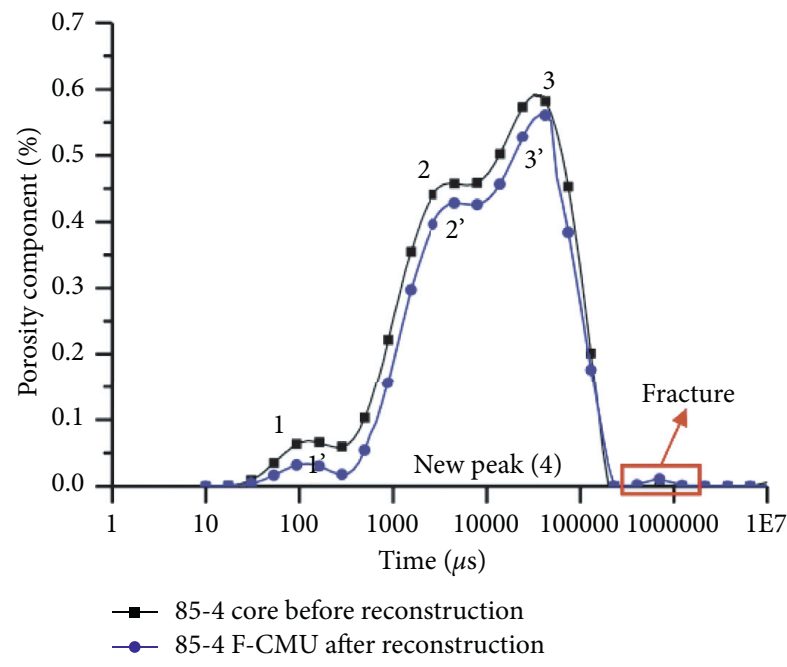

(a)

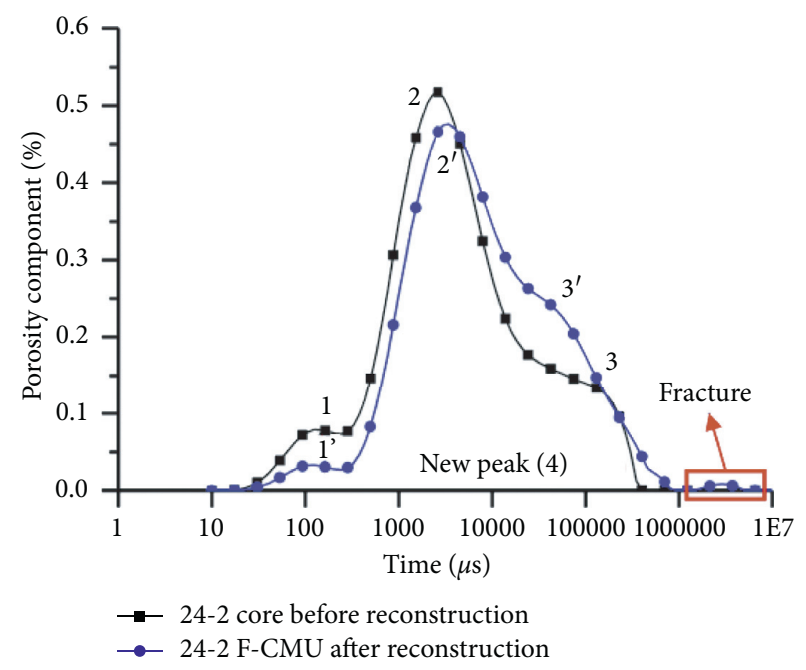

(b)

FiguRE 11: NMR T2 spectra: (a) core 85-4 $\mathrm{T}_{2}$ spectra before and after reconstruction; (b) core 24-2 $\mathrm{T}_{2}$ spectra before and after reconstruction. Note: the marked point is the peak.

$100000-1000000 \mu$ s, respectively, indicating that there are nanoscale pores, micropores, and macropores in it, and peak of No. $1<$ No. $3<<$ No. 2 , indicating that the micropores in 24-2 are developed, with a small number of nanoscale pores and macropores.

After the reconstruction of the F-CMU, the $\mathrm{T}_{2}$ spectra of the core are almost the same as those before the reconstruction, indicating that the core pore structure had almost no change. However, peaks No. 1, No. 2, and No. 3 are slightly smaller than they are before the reconstruction because the pores are compressed and there is pressure in the bonding process, which is consistent with the change in porosity. It is worth noting that after the core is reconstructed, new peaks (No. 4) are observed in both cores in the region greater than $100000 \mu \mathrm{s}$. This region is the embodiment of Y-type and S-type fractures.

3.4. Y-Type and S-Type Fracture Permeability and Numerical Simulation. After the completion of the reconstruction, the permeability of Y-shape and S-shape fractures of F-CMU is measured, respectively. The experimental results are shown in Table 4.
After that, a numerical simulation is carried out on the permeability of Y-type and S-type fractures. The density of $\mathrm{N}_{2}$ gas is calculated according to the ideal gas state equation:

$$
\begin{aligned}
p V & =n R T, \\
\rho & =\frac{m}{V}=\frac{28 p}{[8314 *(273+t)]},
\end{aligned}
$$

where $p$ represents pressure, $V$ represents gas volume, $n$ represents the number of moles of gas, $R$ represents a constant, 8314, $T$ represents thermodynamic temperature, and $\rho$ represents density. At $19^{\circ} \mathrm{C}$ and $25000 \mathrm{~Pa}$, the density of $\mathrm{N}_{2}$ gas is calculated as 0.38 .

The Y-type and S-type fracture models are loaded into the numerical simulation software (COMSOL), and the meshing is selected to refine the mesh. The number of meshes is 435165 (Figure 12).

The simulation results are shown in Figure 13. According to the results, an outlet flow of the Y-type and S-type fractures is obtained, and then the permeability of the fracture is calculated according to Darcy's law as follows: 
TABLE 4: The permeability of the Y-shape and S-shape fractures (experimental results).

\begin{tabular}{lcccccc}
\hline Fracture shape & ID of samples & Temperature $\left({ }^{\circ} \mathrm{C}\right)$ & $\mathrm{N}_{2}$ gas viscosity $(\mathrm{Pa} \cdot \mathrm{s})$ & Inlet pressure $(\mathrm{Pa})$ & Outlet pressure $(\mathrm{Pa})$ & Permeability $(\mathrm{mD})$ \\
\hline $\begin{array}{l}\text { Y-shape } \\
\text { fracture }\end{array}$ & $85-4$ & 19 & 0.016576 & 25000 & 0 & 38854.75 \\
S-shape fracture & $24-2$ & 19 & 0.016576 & 25000 & 0 & 53340.13 \\
\hline
\end{tabular}

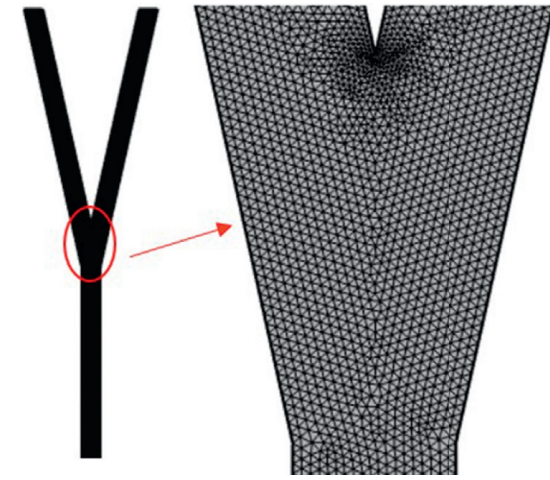

(a)
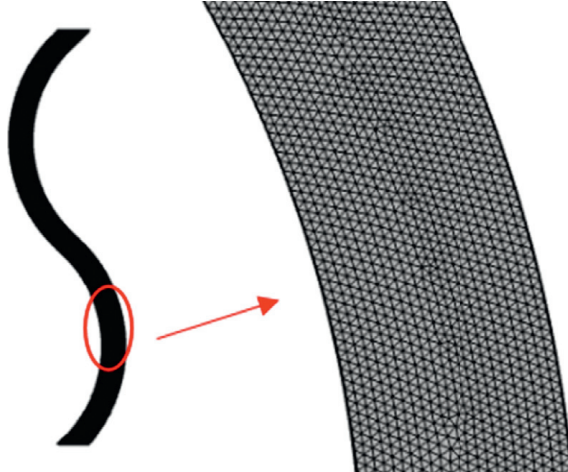

(b)

Figure 12: Model and grid of fracture: (a) Y-shape and (b) S-shape.

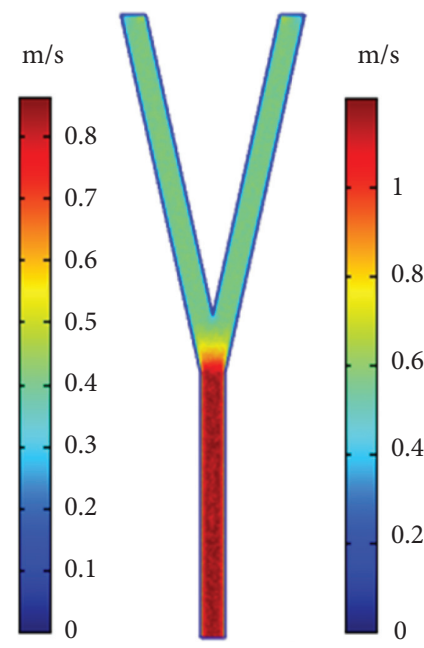

(a)

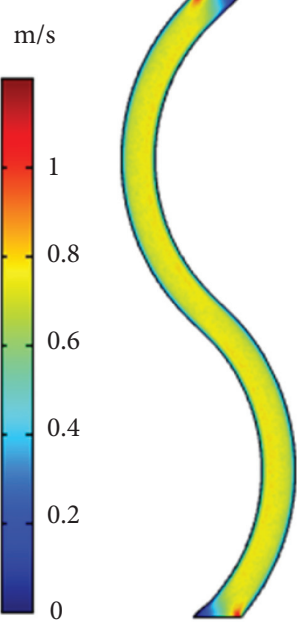

(b)

Figure 13: Velocity field: (a) Y-shape and (b) S-shape.

$$
\begin{aligned}
Q & =\frac{K_{f} A\left(P_{0}-P_{1}\right)}{\mu L}, \\
K_{f} & =\frac{Q \mu L}{A\left(P_{0}-P_{1}\right)},
\end{aligned}
$$

where $Q$ represents outlet flow, $K_{f}$ represents permeability of the fracture, $A$ represents the cross-sectional area, $P_{0}$ represents inlet pressure, $P_{1}$ represents outlet pressure, $\mu$ represents viscosity, and $L$ represents length. The calculation results are shown in Table 5.

The numerical simulation result (Figure 13) shows the velocity field of the Y-type and S-type fractures, indicating that the fluid flow rate in the $\mathrm{Y}$-shaped fracture is very high at the inlet and begins to decrease at the bifurcation. The S-type fracture flow rate is basically equal throughout the process due to a lack of bifurcation. According to the calculation results (Table 5), the two outlet flow rates of the Y-shaped fracture are $0.38294 \mathrm{~cm}^{3} / \mathrm{s}$ and $0.38318 \mathrm{~cm}^{3} / \mathrm{s}$, respectively, and the S-type fracture outlet flow rate is $0.69674 \mathrm{~cm}^{3} / \mathrm{s}$, indicating that the bifurcation of the Y-shaped fracture has a shunting effect. According to Darcy's law, the permeability of Y-type and S-type fractures is calculated to be $38863.83 \mathrm{mD}$ and $53347.92 \mathrm{mD}$, respectively. Compared with the experimental test and numerical simulation results, the fracture permeability has good consistency.

Based on the porosity, permeability, surface wettability, and pore size distribution of the core matrix system before 
TABLE 5: Numerical simulation to calculate permeability results.

\begin{tabular}{lcccc}
\hline Fracture shape & ID of samples & Outlet & Export flow $\left(\mathrm{cm}^{3} / \mathrm{s}\right)$ & Permeability $(\mathrm{mD})$ \\
\hline Y-shape fracture & $85-4$ & 1 & 0.38294 & 38863.83 \\
S-shape fracture & $24-2$ & 2 & 0.38318 & 53347.92 \\
\hline
\end{tabular}

and after reconstruction, it can be concluded that, first, compared with the parallel plate method and splitting reconstruction, our method can realize the transition from single uncertain fracture to more complex bifurcation and tortuous fracture; secondly, compared with chemical dissolution and $3 \mathrm{D}$ printing methods, our method ensures that the mineral composition of the core plug is not changed. Therefore, the advantage of the reconstruction method proposed in this paper is that, on the premise of ensuring that the core is not damaged, the designed fracture closer to the real shape is inserted into the matrix system.

In addition, the permeability of the Y-shaped and S-shaped fractures has about a $10^{4}$ magnitude of matrix permeability. The F-CMU core reconstruction not only reflects the permeability difference between the fracture system and matrix system in a real reservoir but also satisfies the conditions needed for dynamic imbibition experiments at a core scale.

In future research, dynamic imbibition tests of F-CMUs can be carried out using the established Y-type and S-type fractures to study seepage problems coupled with fracture and matrix systems in the F-CMU. In addition, it will also be possible to continue to transform simple fractures into complex fractures to create an F-CMU that is closer to the actual site.

\section{Conclusions}

In this study, a novel method for core reconstruction of F-CMU is proposed. The conclusions are as follows:

(1) Using laser engraving technology and 3D printing materials, a low-damage F-CMU reconstruction technique is proposed, and the correctness and feasibility of the method is verified. The advantage is that the fracture morphology and physical parameters can be designed according to the experimental needs.

(2) The core has a negligible change in matrix porosity, permeability, and surface wettability by experimental test before and after reconstruction, indicating that the proposed reconstruction method does not change the physical properties and structure of the matrix system.

(3) The permeability of the Y-type and S-type fractures is $38854.75 \mathrm{mD}$ and $53340.13 \mathrm{mD}$, respectively, which is about $10^{4}$ orders of magnitude of the matrix system permeability. The established F-CMU satisfies the conditions needed for dynamic imbibition experiments at the core scale. The numerical calculation result has good consistency with the experimental results.

\section{Data Availability}

The data used to support the findings of this study are available from the corresponding author upon request.

\section{Conflicts of Interest}

The authors declare that there are no conflicts of interest regarding the publication of this paper.

\section{Acknowledgments}

This research was funded by the National Science and Technology Major Project of China (grant no. 2017ZX05013006-002).

\section{References}

[1] R. Song, M. Cui, and J. Liu, "Single and multiple objective optimization of a natural gas liquefaction process," Energy, vol. 124, pp. 19-28, 2017.

[2] Z. Y. Wang, Z. M. Yang, Y. H. Ding et al., "A generalized capillary imbibition model for porous media in tight reservoirs," Advances in Civil Engineering, vol. 2018, Article ID 4148734, 8 pages, 2018.

[3] A. S. Abd, E. Elhafyan, A. R. Siddiqui, W. Alnoush, M. J. Blunt, and N. Alyafei, "A review of the phenomenon of counter-current spontaneous imbibition: analysis and data interpretation," Journal of Petroleum Science and Engineering, vol. 180, pp. 456-470, 2019.

[4] Q. Liu, R. Song, J. J. Liu, Y. Lei, and X. Zhu, "Pore-scale visualization and quantitative analysis of the spontaneous imbibition based on experiments and micro-CT technology in low permeability mixed-wettability rock," Energy Science \& Engineering, pp. 1-17, 2020.

[5] J. W. Graham and J. G. Richardson, "Theory and application of imbibition phenomena in recovery of oil," Journal of Petroleum Technology, vol. 11, no. 2, pp. 65-69, 1959.

[6] C. C. Mattax and J. R. Kyte, "Imbibition oil recovery from fractured, water-drive reservoir," Society of Petroleum Engineers Journal, vol. 2, no. 2, pp. 177-184, 1962.

[7] H. Kazemi and L. S. Merrill, "Numerical simulation of water imbibition in fractured cores," Society of Petroleum Engineers Journal, vol. 19, no. 3, pp. 175-182, 1979.

[8] B. J. Bourbiaux and F. J. Kalaydjian, "Experimental study of cocurrent and countercurrent flows in natural porous media," SPE Reservoir Engineering, vol. 5, no. 3, pp. 361-368, 1990.

[9] D. S. Schechter, D. Zhou, and F. M. Orr, "Capillary imbibition and gravity segregation in low IFT systems," Infection \& Immunity, vol. 71, no. 12, pp. 6734-6741, 1991.

[10] J. J. Roberts and W. Lin, "X-ray radiography of fracture flow and matrix imbibition in topopah spring tuff under a thermal gradient," International Journal of Rock Mechanics \& Mining Sciences, vol. 34, no. 3, p. 259, 1997.

[11] Y.-S. Wu, L. Pan, and K. Pruess, "A physically based approach for modeling multiphase fracture-matrix interaction in 
fractured porous media," Advances in Water Resources, vol. 27, no. 9, pp. 875-887, 2004.

[12] Z. T. Karpyn, P. M. Halleck, and A. S. Grader, "An experimental study of spontaneous imbibition in fractured sandstone with contrasting sedimentary layers," Journal of Petroleum Science and Engineering, vol. 67, no. 1-2, pp. 48-56, 2009.

[13] R. Dutta, C.-H. Lee, S. Odumabo et al., "Experimental investigation of fracturing-fluid migration caused by spontaneous imbibition in fractured low-permeability sands," SPE Reservoir Evaluation \& Engineering, vol. 17, no. 1, pp. 74-81, 2014.

[14] J. J. S. de Figueiredo, J. Schleicher, R. R. Stewart, and N. Dyaur, "Estimating fracture orientation from elastic-wave propagation: an ultrasonic experimental approach," Journal of Geophysical Research Solid Earth, vol. 117, no. B8, pp. 1-13, 2012.

[15] J. J. S. Figueiredo, J. Schleicher, R. R. Stewart et al., "Shear wave anisotropy from aligned inclusions: ultrasonic frequency dependence of velocity and attenuation," Geophysical Journal International, vol. 193, no. 1, pp. 475-488, 2013.

[16] L. K. Santos, J. J. S. de Figueiredo, B. Omoboya, J. Schleicher, R. R. Stewart, and N. Dyaur, "On the source-frequency dependence of fracture-orientation estimates from shear-wave transmission experiments," Journal of Applied Geophysics, vol. 114, pp. 81-100, 2015.

[17] J. M. Assad, R. H. Tatham, J. A. Mcdonald, T. M. Kusky, and J. Jech, "A physical model study of scattering of waves by aligned cracks: comparison between experiment and theory1," Geophys, vol. 41, no. 3, pp. 323-339, 1993.

[18] J. M. Assad, J. A. Mcdonald, R. H. Tatham, and T. M. Kusky, "Elastic wave propagation in a medium containing oriented inclusions with a changing aspect ratio: a physical model study," Geophys, vol. 125, no. 1, pp. 163-172, 1996.

[19] J. S. Rathore, E. Fjaer, R. M. Holt, and L. Renlie, "P- and S-wave anisotropy of a synthetic sandstone with controlled crack geometryl1," Geophysical Prospecting, vol. 43, no. 6, pp. 711-728, 1995.

[20] P. Tillotson, J. Sothcott, A. I. Best, M. Chapman, and X.-Y. Li, "Experimental verification of the fracture density and shearwave splitting relationship using synthetic silica cemented sandstones with a controlled fracture geometry," Geophysical Prospecting, vol. 60, no. 3, pp. 516-525, 2012.

[21] P. Tillotson, M. Chapman, A. I. Best et al., "Observations of fluid-dependent shear-wave splitting in synthetic porous rocks with aligned penny-shaped fractures," Geophysical Prospecting, vol. 59, no. 1, pp. 111-119, 2010.

[22] K. Amalokwu, A. I. Best, J. Sothcott, M. Chapman, T. Minshull, and X.-Y. Li, "Water saturation effects on elastic wave attenuation in porous rocks with aligned fractures," Geophysical Journal International, vol. 197, no. 2, pp. 943-947, 2014.

[23] L. K. Santos, J. J. S. de Figueiredo, D. L. Macedo, A. L. de Melo, and C. B. da Silva, "A new way to construct synthetic porous fractured medium," Journal of Petroleum Science and Engineering, vol. 156, pp. 763-768, 2017.

[24] R. Song, J. Liu, and M. Cui, "A new method to reconstruct structured mesh model from micro-computed tomography images of porous media and its application," International Journal of Heat and Mass Transfer, vol. 109, pp. 705-715, 2017.

[25] R. Song, M. Cui, J. Liu, P. G. Ranjith, and Y. Lei, "A pore-scale simulation on thermal-hydromechanical coupling mechanism of rock," Geofluids, vol. 2017, Article ID 7510527, 12 pages, 2017.
[26] R. Song, J. J. Peng, S. Y. Sun et al., "Visualized experiments on residual oil classification and its influencing factors in waterflooding using micro-computed tomography," Journal of Energy Resources Technology, vol. 142, no. 8, pp. 1-15, 2020.

[27] R. R. Stewart, N. Dyaur, B. Omoboya, J. J. S. de Figueiredo, M. Willis, and S. Sil, "Physical modeling of anisotropic domains: ultrasonic imaging of laser-etched fractures in glass," Geophysics, vol. 78, no. 1, pp. D11-D19, 2013.

[28] L. Huang, R. R. Stewart, N. Dyaur, and J. Baez-Franceschi, "3d-printed rock models: elastic properties and the effects of penny-shaped inclusions with fluid substitution," Geophysics, vol. 81, no. 6, pp. D669-D677, 2016.

[29] P. Xu, B. Yu, M. Yun, and M. Zou, "Heat conduction in fractal tree-like branched networks," International Journal of Heat and Mass Transfer, vol. 49, no. 19-20, pp. 3746-3751, 2006.

[30] S. Wang and B. Yu, "Study of the effect of capillary pressure on the permeability of porous media embedded with a fractal-like tree network," International Journal of Multiphase Flow, vol. 37, no. 5, pp. 507-513, 2011.

[31] T. Guo, S. Zhang, Z. Qu, T. Zhou, Y. Xiao, and J. Gao, "Experimental study of hydraulic fracturing for shale by stimulated reservoir volume," Fuel, vol. 128, no. 14, pp. 373-380, 2014.

[32] P. Tan, Y. Jin, K. Han et al., "Analysis of hydraulic fracture initiation and vertical propagation behavior in laminated shale formation," Fuel, vol. 206, pp. 482-493, 2017.

[33] Q. Zheng and B. M. Yu, "Fractal permeability model for gas flow through dual-porosity media," Journal of Applied Physics, vol. 111, no. 2, Article ID 024316, 2012.

[34] F. Bobaru and G. Zhang, "Why do cracks branch? A peridynamic investigation of dynamic brittle fracture," International Journal of Fracture, vol. 196, no. 1-2, pp. 59-98, 2016.

[35] Q. H. Sun, S. L. Chen, and H. Lin, Daqing Toutai Oilfield Development and Construction Collection, Petroleum Industry Press, China National Petroleum Corporation, China, 2008.

[36] G. R. Coates, L. Z. Xiao, and M. G. Prammer, NMR Logging Principles and Applications, Halliburton Energy Services, Gulf Publishing Company, Houston, TX, USA, 1999.

[37] J. Youjun, K. Vafai, and R. Lewis, "Analysis of pore scale fluid migration in a porous medium- application to coal rock seam," International Journal of Numerical Methods for Heat \& Fluid Flow, vol. 27, no. 8, pp. 1706-1719, 2017.

[38] T. Chen, X.-T. Feng, G. Cui, Y. Tan, and Z. Pan, "Experimental study of permeability change of organic-rich gas shales under high effective stress," Journal of Natural Gas Science and Engineering, vol. 64, no. 1, pp. 1-14, 2019.

[39] R. Song, Y. Wang, J. J. Liu et al., "Comparative analysis on pore-scale permeability prediction on micro-CT images of rock using numerical and empirical approaches," Energy Science \& Engineering, vol. 6, no. 7, pp. 2842-2854, 2019.

[40] D. K. Keelan, "Automated core measurement system for enhanced core data at overburden conditions," in Proceedings of the SPE Rocky Mountain Regional Meeting, Billings, Montana, May 1986.

[41] S. C. Jones, "A rapid accurate unsteady klinkenberg permeameter," Society of Petroleum Engineers Journal, vol. 12, no. 5, pp. 383-397, 1972.

[42] Y. Wang, R. Song, J.-J. Liu, M.-M. Cui, and P. G. Ranjith, "Pore scale investigation on scaling-up micro-macro capillary number and wettability on trapping and mobilization of residual fluid," Journal of Contaminant Hydrology, vol. 225, Article ID 103499, 2019. 